\title{
CD34/TK75 Retroviral Vector-Transduced Donor Lymphocytes
}

National Cancer Institute

\section{Source}

National Cancer Institute. CD34/TK75 Retroviral Vector-Transduced Donor

Lymphocytes. NCl Thesaurus. Code C82409.

A preparation of donor T-lymphocytes that are transfected with a retroviral vector encoding a chimeric suicide gene consisting of the extracellular and transmembrane domains of human CD34 and mutant 75 of the herpes simplex virus thymidine kinase (HSV-T K75) with potential controllable immunomodulating activity. Donor T cell therapy following allogeneic hematopoietic stem cell (HSC) transplantation may result in a graftversus-leukemia (GVL) and help control transplant-related viral infections. In the event that graft-versus-host disease (GVHD) develops due to donor lymphocyte infusion, CD34/T K75-transduced donor lymphocytes may be selectively eliminated by administration of the prodrug antiviral agent ganciclovir GCV. In CD34/T 75-transduced donor lymphocytes, GCV is phosphorylated by expressed HSV-T K75 to its monophosphate form and, subsequently, converted into its active triphosphate form, which specifically kills the donor lymphocytes. The expressed CD34 moiety of the chimeric suicide gene serves as a selection marker; mutant 75 of HSV-TK confers increased GCV sensitivity. 